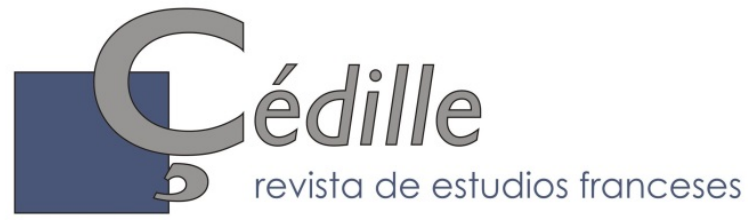

\title{
Linguistique textuelle et graphématique du français en diachronie. Introduction
}

\author{
Elena LLAMAS-POMBO \\ Universidad de Salamanca \\ pombo@usal.es \\ https://orcid.org/0000-0002-8791-3958
}

\section{Le français en diachronie}

Ce numéro monographique de Çédille, revista de estudios franceses rassemble un premier recueil d'articles issus du Colloque International DIACHRO-IX, qui s'est tenu à la Faculté de Philologie de l'Université de Salamanque (Espagne), les 28, 29 et 30 mars $2019^{1}$.

Depuis sa première édition en 2002, le cycle de colloques de la série DIACHRO convoque tous les deux ans la communauté internationale des linguistes et des philologues qui travaillent sur les changements et évolutions que le français a connus de ses origines à nos jours. Il constitue un lieu de réflexion et de discussion autour de recherches novatrices sur les phénomènes du changement linguistique en français, quelles que soient la période considérée et l'approche théorique et méthodologique. Les publications issues des colloques DIACHRO permettent d'accéder à l'état actuel de la recherche en linguistique diachronique $d u$ français, de faire un bilan sur les lacunes et les questions qui restent à examiner en profondeur et de détecter ainsi de nouveaux champs de recherche à développer ou de nouveaux corpus à explorer ${ }^{2}$.

\footnotetext{
${ }^{1}$ Le Colloque DIACHRO-IX et le présent numéro monographique de la revue Çédille ont bénéficié du soutien du projet de recherche FFI2017-84404-P, Énonciation et marques d'oralité dans la diachronie $d u$ français, financé par le Ministerio de Ciencia e Innovación d'Espagne. Cette publication fait partie du Plan quinquennal de recherche de l'Instituto de Estudios Medievales, Renacentistas y de Humanidades digitales (IEMYRhd) de l'Université de Salamanque (Espagne).

${ }^{2}$ Voir dans les Références bibliographiques : (a) Publications issues du cycle de Colloques Internationaux $D I A C H R O$ par date de parution.
} 
La linguistique diachronique du français connait ${ }^{3}$ un intérêt soutenu depuis les dernières décennies. La parution en 2020 de la Grande grammaire historique du français (GGHF, Marchello-Nizia, Combettes, Prévost et Scheer, $2020: 3$, 6) répond à cet intérêt renouvelé pour l'évolution linguistique. Cette " Histoire de la langue française ", envisagée comme une grammaire $d u$ changement, a fait converger et a synthétisé les acquis scientifiques du dernier demi-siècle sur l'évolution de la langue : les avancées théoriques du changement (grammaticalisation, réanalyse, analogie), le développement de la linguistique de corpus et l'exploitation des ressources lexicologiques des grands dictionnaires informatisés.

Le développement de plusieurs domaines d'étude sur le changement de la langue pendant les cinquante dernières années, notamment ceux qui concernent l'énonciation, la pragmatique et le système d'écriture, ont élargi les domaines linguistiques qu'embrasse la Grammaire historique : aux champs canoniques de la phonétique, la morphologie ou la morphosyntaxe, la syntaxe et le lexique, la GGHF adjoint de plein droit les domaines de la graphématique, la sémantique grammaticale, l'énonciation, la pragmatique et la linguistique textuelle.

En particulier, la partie 4 de la GGHF est consacrée au code écrit et à l'histoire des graphies et de la ponctuation du français. "Quel que soit son objet de recherche, les données du linguiste, historien de la langue, transitent par l'écriture, elle est son matériau ", affirment ses auteures, Gabriella Parussa et Yvonne Cazal (2020: 493), dans la présentation des acquis du dernier siècle dans l'histoire de l'orthographe française et dans la linguistique de l'écrit.

Il est également significatif, comme résultat de l'essor de la graphématique historique, que la deuxième édition de la Grammaire du français médiéval de Claude Buridant (2019) s'ouvre sur des «Éléments de graphématique », précédant les "Éléments de phonologie et de morphophonologie ", domaine graphique qui ne faisait pas l'objet d'un chapitre à part dans sa première édition, la Nouvelle grammaire de l'ancien français (Buridant, 2000).

Si la compétence orale des locuteurs a été un objet d'étude majeur de la linguistique synchronique moderne au $\mathrm{XX}^{\mathrm{e}}$ siècle, tout comme l'évolution phonétique avait été longtemps au centre de la recherche en grammaire historique, il n'est pas moins certain que depuis les cinq dernières décennies, la linguistique française a été pionnière dans l'analyse scientifique des systèmes d'écriture et de la compétence écrite des usagers de la langue. Toute une linguistique de l'écrit s'est ainsi développée, se donnant pour objet la description des spécificités du français écrit et de son évolution au cours du temps. Poursuivant la perspective de l'histoire de l'orthographe entreprise par Ferdinand Brunot et par Charles Beaulieux, Nina Catach et l'équipe de recherche qu'elle a

\footnotetext{
${ }^{3}$ Cette Introduction, ainsi que les articles de Soili Hakulinen et de Manuel Bruña Cuevas sont écrits conformément aux rectifications orthographiques de 1990 approuvées par l'Académie française.
} 
dirigée au CNRS ont amplement complété l'histoire graphique de la langue française et ont doté cette histoire d'un ouvrage lexicographique assez exceptionnel dans le panorama de la lexicographie des langues romanes, le Dictionnaire Historique de l'Orthographe française (Catach, 1995b). L'entreprise historique commencée par Catach (1968) s'est accompagnée de l'établissement d'un cadre théorique descriptif, celui du plurisystème graphique du français, applicable aussi bien à la description synchronique qu'à la graphématique diachronique (Catach et al. 1971 ; Catach, 1988, 1994, 1995a, 1998, 2001a, 2001b, etc.).

Dans les dernières décennies du $\mathrm{XX}^{\mathrm{e}}$ siècle, la linguistique française de l'écrit s'instaure ainsi comme discipline autonome à part entière, avec d'autres jalons importants comme les travaux de Claire Blanche-Benveniste et André Chervel (1978 [1969]) et la graphématique autonome de Jacques Anis (1983; Anis et al., 1988). L'histoire de l'orthographe française prend également son essor par son entrée dans l'histoire culturelle avec les essais de Bernard Cerquiglini (1991, 1995, 1996, 2004, 2007, 2018).

Dans le contexte international de la linguistique historique, un intérêt renouvelé est également décelable dans le domaine de l'histoire de l'orthographe. Par exemple, les études recueillies par Marco Condorelli (2020 et à paraitre) sur le développement des systèmes d'écriture en Europe s'intéressent aux nouveaux modèles de description et aux méthodes de recherche empirique qui pourraient enrichir l'histoire de l'orthographe jusqu'à la "refondation » de celle-ci comme discipline indépendante basée sur la comparaison entre plusieurs langues et sur la considération de la standardisation graphique comme un tout, observable dans une perspective interlinguistique. C'est ce même renouveau des approches comparées qui a été retenu comme l'un des trois axes sur lesquels portera le prochain Colloque International DIACHRO-X, Le Français en Diachronie (à célébrer à l'Université de la Sorbonne en 2022), intitulé : L'apport de l'approche comparée des langues romanes à la diachronie du français. Au sein de cet axe, l'émergence d'une graphématique comparée des langues romanes et le transfert de modèles d'analyse entre les philologies des différents pays se présentent comme des domaines de recherche encore à développer ( $c f$. Condorelli et Voeste, 2020 et LlamasPombo, 2020a).

Nous estimons également pertinent de citer ici l'apport de la graphématique française à la graphématique diachronique hispanique, à travers un transfert de modèles d'analyse qui augure de nouveaux enrichissements. Cabrera (1998), Carrera de la Red (1998) ou Sánchez-Prieto Borja (2012) ont souligné l'intérêt de développer une scriptologie des scriptas hispaniques médiévales, analogue à celles des scriptologies du domaine gallo-roman ou du domaine italo-roman. La théorie linguistique de la ponctuation de Nina Catach (1980, 1994 ; Catach et al. 1977 et 1979, etc.) a également servi de modèle pour l'analyse diachronique de la séquenciation des mots en espagnol (Llamas-Pombo, 2009) et se trouve présente dans les nouveaux apports à l'histoire de la ponctuation en espagnol ( $c f$., par exemple, Las Heras Calvo, 2020 : 97-105). 


\section{Linguistique textuelle diachronique du français}

Les phénomènes textuels en diachronie constituent un domaine qui avait été peu étudié avant le XXI" siècle (Combettes, 2012 : 4 ; Combettes 2019 et Capin et al., 2019 : XV). Afin de combler ce vide, Bernard Combettes (2012) a déterminé les conditions théoriques et méthodologiques pour que se constitue en discipline spécifique la linguistique textuelle diachronique, au même titre que la phonétique historique (qui a énoncé des lois d'évolution), que la lexicologie (consacrée à déceler les règles de changement de sens) ou que la syntaxe, la morphologie ou la sémantique historiques. Combettes a ainsi établi un programme de valorisation de la recherche diachronique dans la linguistique $d u$ texte, conçue comme une étude du système de la langue prenant en compte la dimension discursive ; une linguistique reposant sur la notion de "codage " et s'appuyant obligatoirement sur l'observation des relations qui s'établissent entre les marques fournies par le système linguistique à un moment donné et les catégories textuelles. L'étude des principes linguistiques qui signalent la cohérence discursive et ses marques, ainsi que la hiérarchisation des énoncés devrait se trouver au premier plan de cette discipline émergente. Une discipline attentive à la compétence textuelle des locuteurs au cours du temps, aux modes de lecture et de production des textes écrits et, par conséquent, aux rapports entre langue et cognition (Combettes, $2012: 4$ ).

En 2012, il était encore difficile - comme l'affirmait Combettes - de concevoir un " manuel de linguistique textuelle historique », comme il en existe pour la " phonétique historique", par exemple. Or cette discipline prend actuellement son essor, comme le prouve la présence d'un chapitre sur la cohérence discursive et les unités pertinentes au niveau informationnel au sein de la Grande Grammaire Historique du Français (GGHF et Combettes 2020). Le présent numéro monographique inclut quatre contributions à cette étude émergente du domaine discursif.

Céline Guillot décrit les chaines de référence (les mentions successives d'une même entité de discours), dans le prolongement de travaux antérieurs dans ce domaine (notamment deux numéros des revues Langages et Langue française, cf. Schnedecker, 2014 et Schnedecker et al., 2017). Guillot décrit les chaines de référence dans un corpus de textes narratifs écrits entre le début du XII ${ }^{e}$ et la fin du XV $\mathrm{XV}^{\mathrm{e}}$ siècle, à l'aide d'une numérisation annotée et d'une série de mesures, qui portent sur la couverture des textes par les chaines, la taille de celles-ci, leurs référents et leurs catégories grammaticales (réalisées grâce à la plateforme d'analyse TXM). L'objectif en est de détecter des corrélations entre le matériau des chaines et l'évolution des modes de structuration textuelle et des systèmes de division interne. Au-delà des conclusions sur le corpus étudié, l'intérêt de cet article tient au fait qu'il contribue à « inventer une méthodologie » et à " affiner la méthode » de description des chaines à l'aide des outils numériques.

L'article de Gilles Siouffi constitue un apport spécifique à la diachronie des pratiques d'écriture, centré sur la question de la séquencialité discursive. Cette contribution va dans le sens d'une linguistique textuelle diachronique, tout en posant des 
questions à développer, ainsi qu'un cadre conceptuel favorisant de futures recherches sur les modèles de séquencialité (le lecteur trouvera ainsi une définition des concepts de séquencialité de l'écrit, textualité, médialité (contraintes et affordances) et littéracie ou syntactisation). Siouffi pose la pertinence des notions de phrase et de période pour aborder la séquencialité d'un texte, tout en faisant intervenir l'analyse de la "logique " de la ponctuation originelle des textes des $\mathrm{XVII}^{\mathrm{e}}$ et $\mathrm{XVIII}{ }^{\mathrm{e}}$ siècles. Au-delà des pratiques de ces deux siècles, la période, pourrait-elle constituer un outil "transchronique " appliquable à n'importe quelle production discursive ? Quel a été le rôle de la ponctuation dans la définition traditionnelle de la notion de phrase par ses limites? Si la phrase n'est pas la seule forme de séquentialité en diachronie, à travers trois études de cas, Siouffi met en lumière l'existence de segmentations ou d'associations larges qui ne sont pas non plus des périodes au sens classique, mais des assemblages qui n'ont pas de configuration particulière, faisant sens, toutefois, au niveau sémantique et discursif. Pour ne citer que l'un des prolongements possibles de l'apport théorique de cet article, son application à l'analyse de la séquencialité de l'écrit documentaire médiéval pourrait éclairer comment fonctionnent textuellement les chartes qui ne se laissent pas analyser à partir d'une syntaxe uniquement phrastique.

Claire Badiou-Monferran et Daniéla Capin proposent trois études de cas dédiées aux coocurrences de et + adverbe en diachronie longue (et aussi / et également; et puis / et après; et plus / et en plus / et de plus), à partir du corpus linguistique Frantext. Elles font appel au cadre théorique de la transcatégorisation (ou variation de catégorie grammaticale ou sémantique d'une même forme linguistique), tout en élargissant ce concept en tant que type de changement linguistique. Les résultats de l'observation des coocurrences mettent en lumière une chronologie et un parcours historique différents pour chaque combinaison. Mais ces résultats éclairent aussi un phénomène ouvert à la recherche : "celui de l'autonomisation du code écrit par rapport au code oral, et de son influence sur la configuration des termes qui agissent sur l'organisation textuelle ". L'analyse de la ponctuation en diachronie est également convoquée dans cette étude sur l'organisation textuelle.

Mathieu Goux propose, comme introduction à son étude, une ample réflexion épistémologique sur les enjeux de la grammaire textuelle, ainsi qu'une définition textuelle des connecteurs; deux contributions théoriques qui pourront éclairer de futures recherches. L'auteur analyse l'évolution de deux connecteurs additifs (puis et ensuite) et trois connecteurs adversatifs (cependant, néanmoins, pourtant) en français classique. Leur rôle est étudié en rapport avec la ponctuation et avec la dynamique informationnelle des énoncés. Les résultats de cette analyse montrent que le rôle textuel des connecteurs a accompagné le passage de l'écriture périodique à l'écriture phrastique, en donnant aux usagers de la langue de nouvelles ressources d'organisation des propos.

Soili Hakulinen s'intéresse à l'évolution de la textualité en français entre le XIV et le $\mathrm{XX}^{\mathrm{e}}$ siècle, à travers l'étude des jonctions interpropositionnelles, dans un corpus 
parallèle mis en contraste : d'une part, une traduction française anonyme du texte latin de la Consolatio Philosophiae de Boèce (VI ${ }^{\mathrm{e}}$ siècle), conservée dans un manuscrit de la fin du XIV ${ }^{e}$ siècle ; d'autre part, la traduction moderne de Bocognano (1937) du même texte latin. Sont ainsi comparés deux états du français; celui du XIV ${ }^{e}$ siècle, où les conventions de la langue écrite n'étaient pas encore standardisées, et celui du français moderne, à l'époque où l'influence d'Internet ne s'est pas encore fait sentir dans les conventions d'écriture. Hakulinen examine trois facteurs contribuant au degré d'intégration des textes: la dépendance syntaxique, l'emploi de joncteurs et la ponctuation. La théorie des jonctions interpropositionnelles de Raible (1992) permet à cet auteur d'examiner dans les deux traductions les manières de relier les propositions entre elles et leur contribution au tissage textuel. Le contraste dans les taux de ponctuation entre la traduction du XIV ${ }^{\mathrm{e}}$ et celle du XX $\mathrm{XX}^{\mathrm{e}}$ siècle est net : le texte ancien présente une préférence à marquer la jonction par un joncteur plutôt que par un signe de ponctuation, tandis que le nombre total des propositions ponctuées est en effet bien plus élevé dans la traduction moderne. Ces résultats convergent avec les études statistiques menées sur des corpus numérisés de français contemporain, qui ont permis de vérifier de façon quantitative l'évolution de la syntaxe, de la ponctuation et de la corrélation entre les deux. Pour Brunet (1999 : 713), concrètement, si la phrase est complexe, elle n'aura pas la même segmentation qu'une phrase simple, et le dosage des virgules et des points sera différent. La statistique a confirmé que les constructions phrastiques ont tendance à se simplifier au $\mathrm{XX}^{\mathrm{e}}$ siècle : "si l'on définit grossièrement la phrase comme l'espace textuel compris entre deux points, on voit que cet espace s'est réduit au cours des siècles" (Brunet 1999 : 714). Ce raccourcissement de la phrase a entrainé, pour Brunet, une hausse dans la quantité de signes de ponctuation forte : le point, le point d'exclamation, le point d'interrogation, les points de suspension, les parenthèses et les tirets ont augmenté leur fréquence au $\mathrm{XX}^{\mathrm{e}}$ siècle, en tant que marques de la segmentation croissante du discours.

La détermination des spécificités linguistiques de la langue parlée et de la langue écrite est un domaine de recherche en plein essor ; concrètement, les études sur l'oral représenté en diachronie (Marchello-Nizia, 2014) dans les textes écrits constituent un domaine connexe à la linguistique textuelle. Nous incluons ainsi dans cette première section du présent numéro l'article de Pierre Vermander, dans la mesure où il apporte une réflexion originale sur la dialectique des rapports oral-écrit à partir de textes médiévaux. L'auteur propose une tentative de définition des marqueurs d'oralité à partir de deux prémisses : la considération de l'oral et de l'écrit comme des technologies et non pas comme des codes et la prise en compte d'un imaginaire des systèmes de signes présent dans les anciens textes écrits.

\section{Philologie et graphématique}


L'ecdotique, en tant que cadre méthodologique de l'édition du patrimoine écrit du passé à travers les différents témoins ou variantes d'une même source est le domaine de réflexion par excellence sur la variance linguistique et, spécifiquement, sur les variantes graphiques; philologie et graphématique convergent dans l'interprétation des œuvres anciennes. On sait que «tous les éditeurs de textes médiévaux s'accordent sur la nécessité d'un certain degré de modernisation et des graphies et de la ponctuation " dans les éditions où ils donnent à lire les textes au lecteur contemporain ; or "l'édition ne peut pas refléter la totalité des usages graphiques ", "à moins de devenir une pure transcription paléographique ». La pratique ecdotique dérobe à la lecture des réalités telles que les variantes graphiques, les traditions particulières de séquenciation des mots, la ponctuation et la mise en page des témoignages anciens. "L'édition critique se doit de les reléguer au domaine du marginal, du résiduel » (nous citons une réflexion antérieure sur les implications de la triade ponctuer-éditer-lire, Llamas-Pombo 2008 : 132). Ces réalités en quelque sorte "résiduelles " pour l'édition critique, nous informent néanmoins sur l'ancrage des textes dans leurs circonstances matérielles et temporelles. Leur étude en tant que matériau linguistique et graphique significatif nous rend des informations précieuses sur l'histoire graphique du français, sur l'histoire de la configuration de la page et des modalités de lecture et sur l'histoire génétique ou stemma d'une œuvre, comme le prouve la contribution d'Andrea Valentini au présent numéro monographique. La Philologie matérielle ou Nouvelle philologie fait l'éloge de la variante (selon la formule de Bernard Cerguiglini, 1989) depuis deux décennies, et l'histoire de l'orthographe du français en a tiré profit.

L'article d'Andrea Valentini explore l'étude systématique d'une série de morphèmes et de variantes graphiques dans trois manuscrits tardifs du Livre de la cité des dames de Christine de Pizan, datés dans la première moitié du XV $\mathrm{XV}^{\mathrm{e}}$ siècle et contenant une version remaniée anonyme du Livre de Pizan. Sont ici analysés les éléments suivants : le remplacement de mie par pas; le remplacement de comment par comme; le remplacement de tout soit par tant soit; les variantes graphiques du suffixe -ore /-oire et les variantes du passé simple du verbe avoir ot / olt / eust. L'analyse minutieuse des données permet à l'auteur de prouver que la variation graphique n'est pas « anecdotique " ou " résiduelle ", mais qu' elle fournit trois types d'informations : a) des données pertinentes pour l'histoire graphique du français ; b) des éléments formels significatifs pour affiner la datation et la localisation géographique de différents manuscrits d'une même œuvre ; c) des pistes pour une meilleure compréhension de notre part de la compétence linguistique des scripteurs médiévaux et de leur façon de travailler dans leur labeur de transmission de la langue écrite.

Effectivement, les variantes de graphie et de ponctuation nous informent sur la compétence linguistique des anciens scripteurs du français et sur la façon dont les textes se sont insérés dans la culture humaine de leur temps. C'est ainsi que lire de nos jours les mêmes signes manuscrits (graphies et signes de ponctuation) qui aidaient les lecteurs 
des textes du Moyen Âge, "c'est une façon de redonner du pouls et du présent au livre médiéval », comme nous avons tenté de le démontrer dans une étude antérieure (Llamas-Pombo, $2016: 140$ ).

L'article de Laura-Maï Dourdy et de Michela Spacagno constitue une nouvelle et remarquable démonstration de l'éloquence des variantes, un éloge de la variante dans le domaine de la ponctuation. Faisant écho à l'aspect poétique qu'Henri Meschonnic a attribué au respect de la ponctuation originale des textes, les auteures évoquent le "rythme " et " le pouls" de la langue que véhiculent les signes de ponctuation, ainsi que la tâche d' " auscultation » des signes que se donne le linguiste. En effet, au dire de Meschonnic (2000 : 292), la ponctuation consiste dans «la mise en scène d'une temporalité ». La contribution de ces auteures au présent numéro monographique trouve son point de départ méthodologique dans deux travaux d'édition critique antérieurs, qui ont fait l'objet de deux thèses doctorales : une mise en prose de Jourdain de Blaves et le Mystère de la vie de sainte Marguerite, respectivement. C'est ainsi que l'analyse de la variance graphique sur le plan de la ponctuation surgit d'une réflexion antérieure sur les méthodes ecdotiques. Les auteures étudient dans cet article l'évolution des pratiques de ponctuation à la Renaissance, à partir de cinq traditions textuelles imprimées au $\mathrm{XVI}^{\mathrm{e}}$ siècle, couvrant deux genres différents (trois mystères et deux mises en prose). Elles démontrent empiriquement que les pratiques de ponctuation des imprimeurs étaient d'abord très similaires à celles des scribes médiévaux ; ensuite, que le rôle des imprimeurs, après 1540, sera perceptible dans la fixation des usages. Ce travail offre tout un modèle d'étude de la ponctuation pour cette étape déterminante dans l'histoire de la culture écrite qu'est le passage du manuscrit à l'imprimé : sont prises en compte des variables comme celles qui opposent vers et prose ou les variables de genre littéraire ; la ponctuation comme marque visuelle de la structure syntaxique et énonciative des textes; l'adéquation du fond et de la forme; la variation paléographique ou typographique et les implications graphétiques de la morphologie des signes graphiques et leur signification; enfin, les implications des signes sur les pratiques de lecture. Tout un modèle d'analyse, apte à éclairer de nouveaux corpus et prêt à être élargi et enrichi par le concours de la bibliographie matérielle et l'histoire du livre.

La contribution de Yunhao Na, "Éloge de la pause ", s’inscrit dans le même champ d'étude que les deux articles antérieurs : la comparaison des variantes de ponctuation entre les différentes copies manuscrites ou les imprimés d'une œuvre, comme complément et contribution à l'établissement de sa généalogie ou stemma. Le corpus est formé par six manuscrits et quatre imprimés des récits hagiographiques connus en français sous le titre de Les Festes Nouvelles, datant du XIV ${ }^{\mathrm{e}}$ au XVI ${ }^{\mathrm{e}}$ siècle. Le catalogue des signes employés dans ce corpus est intéressant pour l'étude de la diachronie des signes de ponctuation du français pré-standardisé. L'analyse de la segmentation graphique matérialisée par la ponctuation met en évidence une tendance des typographes à segmenter les textes d'avantage par rapport aux traditions manuscrites; l'article de 
Yunhao Na souligne - tout comme la contribution de Soili Hakulinen - le rapport étroit entre la ponctuation et la diachronie de la textualité du français.

\section{Lexicographie et histoire de l'orthographe}

Les trois articles antérieurs, centrés sur la l'hétérographie caractéristique des manuscrits médiévaux, ont donc souligné l'intérêt des variantes graphiques pour l'histoire de la graphie médiévale. Une certaine variabilité caractérise également le français écrit à partir du XVI e siècle, mais une telle variabilité est dès lors en partie déterminée par différents courants de la pensée grammaticale et ortho-typographique : les grands courants orthographiques du XVII siècle (Biedermann-Pasques, 1992) reflètent l'opposition entre le système graphique " ancien » et les pratiques " modernisées".

Manuel Bruña Cuevas, auteur d'une vaste contribution à l'historiographie linguistique du français et, en particulier, à l'histoire de la lexicographie bilingue françaisespagnol et espagnol-français, fait état dans son article des usages orthographiques adoptés pour le français dans les dictionnaires français-espagnol et espagnol-français publiés de la fin du XVI è la fin du XVIII ${ }^{e}$ siècle, autour de l'opposition entre les " anciennes " et les "nouvelles " pratiques. Cette contribution propose un parcours à travers la variance graphique du français dans les dictionnaires et reconstruit minutieusement l'évolution des conceptions orthographiques dans la lexicographie bilingue entre le XVII ${ }^{\mathrm{e}}$ et le XVIII ${ }^{\mathrm{e}}$ siècle.

\section{Modèles graphématiques et ponctuation}

S'il est un champ de recherche qui a particulièrement contribué à la graphématique historique comme discipline indépendante mais toujours connexe à la phonologie, à la philologie, à la poétique du rythme et à la lecture, c'est celui de l'histoire de la ponctuation comme composante essentielle du système graphique du français. Cette histoire est dotée dès les années 1970 de cadres théoriques et de modèles d'analyse dont les principales contributions françaises sont celles de Nina Catach (1980, 1994, Catach et al. 1977 et 1979, etc.) et de Jacques Anis (1983, Anis et al. 1988, etc.). Ce cadre a permis l'élaboration de plusieurs thèses doctorales sur la spécificité de la ponctuation médiévale (Lavrentiev, 2009 ; Llamas-Pombo, 1996 ; Mazziotta, 2009), ainsi qu'un nombre considérable d'articles spécialisés et de remarquables travaux de synthèse devenus déjà incontournables dans le domaine (notamment, ceux de Maria Careri, 2008 et de Maria Colombo, 2008).

Plusieurs numéros monographiques de revues et des livres collectifs témoignent de l'intérêt pluridisciplinaire actuel pour la ponctuation. Sans prétendre à l'exhaustivité, on peut rappeler Dürrenmatt (2000), L'information grammaticale (Pétillon, 2004), Langue française (Favriaud, 2011), Le Français d'aujourd'hui (David et al., 2014), Littératures (Bikialo et Rault, 2015), Linx (Bikialo et Rault, 2017) et Ferrari et al. (2017), ainsi que les ouvrages issus de différents colloques (Dauvois et Dürrenmatt, 
2011 ; Fasseur et Rochelois, 2016 ; Pétillon et. al. 2016) et les bibliographies sur la ponctuation (Baddeley, 2011 ; Llamas-Pombo, 2008), sans oublier les prolongements théoriques du modèle de Jacques Anis (Dahlet, 2003). La Grande Grammaire Historique du Français (GGHF) accueille l'histoire de la ponctuation au sein de la diachronie du français par l'inclusion d'un chapitre ( $n^{\circ}$ 28) qui propose un modèle théorique ample, appliquable à de futures études diachroniques sur la ponctuation des textes (conceptualisations de Catach, Tournier et Anis, principalement), ainsi qu'une synthèse chronologique sur les signes de ponctuation en français (Llamas-Pombo, 2020b).

Les sections antérieures du présent numéro de la revue Çédille offrent des articles qui traitent de la valeur linguistique des signes de ponctuation pour la textualité écrite (Siouffi, Badiou-Monferran et Capin, Goux, Hakulinen) et pour la tâche de l'édition des textes (Valentini, Dourdy et Spacagno, $\mathrm{Na}$ ). Les trois articles suivants proposent des résultats de recherche en histoire de la ponctuation, tout en apportant de nouveaux modèles d'analyse, de nouvelles méthodes ou de nouvelles perspectives, qui tentent d'élargir le champ d'observation de la compétence scripturaire des usagers du français au cours du temps.

La contribution d'Alexei Lavrentiev démontre que la linguistique quantitative et l'exploitation de bases de données numériques est un impératif incontournable pour l'observation objective des pratiques de ponctuation anciennes. Si la lecture, pour ainsi dire, « linéaire » des textes médiévaux par le linguiste actuel a constitué la source essentielle de la description des tendances graphiques en diachronie, l'approche quantitative développée par Lavrentiev ouvre de nos jours une voie d'analyse qui permet d'affiner les requêtes. C'est ce que prouve cet article centré sur les marques graphiques et lexicales de l'oral représenté dans un ample corpus de textes du $\mathrm{XI}^{\mathrm{e}}$ au XVI $\mathrm{XV}^{\mathrm{e}}$ siècle.

Nicolas Mazziotta pose une question complémentaire et délicate concernant notre observation de la ponctuation médiévale en tant que linguistes. Il s'agit de la nécessité d'expliquer l'absence de ponctuation dans certains manuscrits médiévaux, un fait résultant de la variabilité intrinsèque à la graphie du Moyen Âge. Cet article met en évidence l'existence d'unités non ponctuables, dont les règles d'occurrence devraient être interprétées par le linguiste, au même titre que les unités ponctuables ou contextes ponctuables (selon la terminologie de Lavrentiev, 2009). Les modèles d'étude de la ponctuation médiévale pourront ainsi inclure un questionnement sur l'existence d'une variable ponctuation / marque zéro.

Comme le soulignent Condorelli et Voeste (2020) dans un travail cité antérieurement, une histoire contrastive des orthographes est envisageable et peut contribuer à mieux connaitre la culture écrite de différentes époques. Par exemple, l'ouvrage collectif intitulé Orthographies in Early Modern Europe (Baddeley et Voeste, 2012) est l'une des contributions à cette histoire culturelle de l'orthographe dans l'Europe du $\mathrm{XVI}^{\mathrm{e}}$ siècle. L'article de Juhani Härmä nous offre une étude de cas qui met en œuvre cette perspective d'une graphématique comparée et qui illustre la présence d'interférences 
orthographiques entre les usagers de deux langues (analogues à celles qui se produisent aux niveaux phonique ou lexical dans le contact entre les langues). Ce travail met en lumière les variantes graphiques manuscrites de l'écriture privée, à travers une étude de l'emploi du tiret dans un corpus de correspondances entre quatre épistoliers finlandais de la fin du XVIII et du début du XIX ${ }^{\mathrm{e}}$ siècle. La situation de contact entre langues se produit entre scripteurs de langue maternelle suédoise qui écrivent des lettres en français ou dans les deux langues, situation qui a favorisé une influence du suédois sur le français « langue étrangère ».

\section{Remerciements}

Les éditrices du présent numéro monographique remercient le Département de Philologie Française, la Faculté de Philologie et l'Institut d'études médiévales, de la Renaissance et des humanités digitales (IEMYRhd) de l'Université de Salamanque (Espagne) pour leur financement et pour leur soutien à la tenue du Colloque DIACHRO$I X$, dont est issu ce recueil d'articles.

Nous remercions également les membres du Comité scientifique du Colloque DIACHRO-IX d'avoir apporté leur expertise pour la relecture des contributions :

Susan Baddeley (Université de Versailles Saint-Quentin en Yvelines)

Claire Badiou-Monferran (Université de Lorraine)

Anne Carlier (Université de Lille)

Daniéla Capin (Université de Strasbourg)

Yvonne Cazal (Université de Caen)

Maria Colombo-Timelli (Université de Paris-Sorbonne)

Bernard Combettes (Université de Lorraine)

Corinne Denoyelle (Université Grenoble Alpes)

Benjamin Fagard (CNRS, ENS \& Université Sorbonne Nouvelle Paris 3)

Julie Glikman (Université de Strasbourg)

Sonia Gómez-Jordana Ferary (Universidad Complutense de Madrid)

Céline Guillot-Barbance (École Normale Supérieure de Lyon)

Dominique Lagorgette (Université de Savoie)

Alexey Lavrentiev (École Normale Supérieure de Lyon)

Elena Llamas-Pombo (Universidad de Salamanca)

Christiane Marchello-Nizia (École Normale Supérieure de Lyon)

Sophie Marnette (University of Oxford)

Nicolas Mazziotta (Université de Liège \& Universität Stuttgart)

Evelyne Oppermann-Marsaux (Université Sorbonne Nouvelle Paris 3)

Gabriella Parussa (Université Sorbonne Nouvelle Paris 3)

Sophie Prévost (CNRS, ENS \& Université Sorbonne Nouvelle Paris 3)

Thomas M. Rainsford (Universität Stuttgart)

Amalia Rodríguez Somolinos (Universidad Complutense de Madrid)

Marta Saiz Sánchez (Universidad Complutense de Madrid)

Gilles Siouffi (Université de Paris-Sorbonne) 
Lene Schøsler (Købnehavns Universitet)

Achim Stein (Universität Stuttgart)

Que nos collègues du Comité d'organisation, Amalia Rodríguez Somolinos, Sonia Gómez-Jordana Ferary et Marta Saiz Sánchez, de l'Universidad Complutense de Madrid, soient aussi remerciées pour leur conseil scientifique. Nous tenons également à mentionner l'appui des organisateurs du Colloque DIACHRO-VIII, de l'Université de Strasbourg, pour la célébration du Colloque DIACHRO IX à l'Université de Salamanque.

Nous exprimons également notre gratitude envers le Conseil de rédaction de la revue Çédille pour avoir accepté la publication du présent numéro monographique et pour sa diffusion en libre accès (la revue Çédille est présente dans un grand nombre de bases de données bibliographiques et bibliométriques internationales). Nous remercions spécialement la revue de nous avoir permis un certain nombre de caractéristiques ortho-typographiques, exceptionnelles par rapport aux normes de style éditorial de la revue : le gras a été employé dans la citation des exemples de langue afin de mettre en relief des usages que l'opposition romain/italique ne pouvait pas distinguer; l'emploi de lettres en différentes couleurs a également été nécessaire pour visualiser certaines distinctions linguistiques, tout en faisant l'économie d'autres caractères moins lisibles; dans les références bibliographiques, enfin, nous avons distingué deux sections : l'une pour informer sur les corpus d'étude, l'autre pour inclure les références des études citées.

Finalement, nous tenons à remercier le directeur de la revue Çédille, le professeur José M. Oliver Frade, du soin porté à l'édition des articles, dont l'orthotypographie était particulièrement complexe. Merci d'avoir traité nos données avec la même vertu et sous la même devise des grands typographes humanistes: Festina lente.

\section{RÉFÉRENCES BIBLIOGRAPHIQUES}

a) Publications issues du cycle de Colloques Internationaux DIACHRO, par date de parution

COMBeTtes, Bernard, Christiane MARChello-Nizia \& Sophie PRÉVOST [coord.] (2003) : Verbum, $25: 3$ et 4 [La grammaticalisation en français].

COMBETTES, Bernard \& Christiane MARCHELlO-Nizia [éds] (2007) : Études sur le changement linguistique en français. Nancy, Presses Universitaires de Nancy.

FAGARD Benjamin, Sophie PRÉvost, Bernard COMBETTES \& Olivier BERTRAND [éds] (2008): Évolutions en français. Études de linguistique diachronique. Bern/Berlin / Bruxelles, Peter Lang.

Combettes, Bernard, Céline Guillot, Evelyne Oppermann-Marsaux, Sophie Prévost \& Amalia RODRÍGUEZ SOMOLINOS [éds] (2010) : Le changement en français. Études de linguistique diachronique. Bern / Berlin / Bruxelles, Peter Lang. 
Guillot, Céline, Bernard Combettes, Alexei Lavrentiev, Evelyne OpPermann-MarSAUX \& Sophie PRÉVOST [éds] (2012) : Le changement en français. Études de linguistique diachronique. Bern / Berlin / Bruxelles, Peter Lang.

CARLIER, Anne, Michèle GOYENS \& Béatrice LAMIROY [éds] (2015) : Le français en diachronie. Nouveaux objets et méthodes. Bern / Berlin / Bruxelles, Peter Lang.

PRÉVOST, Sophie \& Benjamin FAGARD [éds] (2017) : Le français en diachronie. Dépendances syntaxiques, morphosyntaxe verbale, grammaticalisation. Bern / Berlin / Bruxelles, Peter Lang.

PARUSSA, Gabriella, Maria COLOMBO TiMELli \& Elena LlAMAS-POMBO [éds] (2017) : Enregistrer la parole et écrire la langue dans la diachronie du français. Tübingen, Narr Francke Attempto (ScripOralia, 143).

CAPIN, Daniéla, Julie GLIKMAN, Vanessa OBRY \& Thierry REVOL [éds] (2019). Le français en diachronie (Moyen français - Segmentation des énoncés - Linguistique textuelle). Strasbourg, EliPhi, Éditions de Linguistique et de Philologie (Travaux de Linguistique romane. Morphologie, syntaxe, grammaticographie).

\section{b) Études citées}

ANIS, Jacques (1983) ; «Pour une graphématique autonome ». Langue française, 53 [J. Anis (éd.), Le signifiant graphique], 31-44.

ANIS, Jacques ; Jean-Louis CHISS \& Christian PUECH (1988) : L'Écriture: théories et descriptions. Bruxelles, De Boeck Université.

BADDELEY, Susan (2011) : " Sources pour l'étude de la ponctuation française au XVI" siècle ", in Nathalie Dauvois \& Jacques Dürrenmatt (éds). La Ponctuation à la Renaissance. Paris, Garnier, 191-227.

BADDELEY, Susan \& Anja VOesTe [éds] (2012) : Orthographies in Early Modern Europe. Berlin / Boston, Walter de Gruyter.

BEAULIEUX, Charles (1927) : Histoire de l'orthographe française. 2 vols. Paris, Champion.

BIEDERMANN-PASQUES, Liselotte (1992) : Les grands courants orthographiques au XVII siècle et la formation de l'orthographe moderne. Tübingen, Max Niemeyer Verlag (Beihefte zur Zeitschrift für Romanische Philologie, 245).

BIKIALO, Stéphane \& Julien RAULT [éds] (2015) : Littératures, 72 [Imaginaires de la ponctuation dans le discours littéraire (fin XIXe siècle-début XXI siècle].

BIKIALO, Stéphane \& Julien RAULT [éds] (2017) : Linx 75 [Imaginaires de la ponctuation. Ordre et inquiétude du discours].

BlanCHE-BENVENISTE, Claire \& André CHERVEL (1978 [1969]) : L'orthographe. Paris, Maspiero.

BRUNET, Étienne (1999) : "La langue française au XX ${ }^{\mathrm{e}}$ siècle. II. Ce que disent les chiffres ", in Jacques Chaurand (éd.) Nouvelle histoire de la langue française, Paris, Seuil, 673727.

BRUNOT, Ferdinand (1905-1938) : Histoire de la langue française. 11 tomes. Paris, A. Colin. 
BURIDANT, Claude (2000) : Grammaire nouvelle de l'ancien français. Paris, Sedes.

BURIDANT, Claude (2019) : Grammaire du français médiéval (XIe - XIVe siècles). Strasbourg, ELiPhi, Éditions de linguistique et de philologie.

CABRERA, Carlos (1998) : «Reflexiones sobre el sistema gráfico avulgarado de los textos primitivos leoneses», in José Manuel Blecua, Juan Gutiérrez \& Lidia Sala (éds). Estudios de grafemática en el dominio hispano. Salamanca, Universidad de Salamanca / Instituto Caro y Cuervo, 9-23.

CAPIN, Daniéla, Julie GlikMAn, Vanessa ObRY \& Thierry ReVOL (2019) : "Présentation ", in Le français en diachronie (Moyen français - Segmentation des énoncés - Linguistique textuelle). Strasbourg, EliPhi, Éditions de Linguistique et de Philologie (Travaux de Linguistique romane. Morphologie, syntaxe, grammaticographie), XI-XVIII.

CARERI, Maria (2008) : « La punteggiatura in Francia. I. Manoscritti provenzali e francesi : dalle orrigini alla fine del XIII secolo ", in Bice Mortara Garavelli (dir.), Storia della punteggiatura in Europa. Roma, Barbi, 213-232.

CARRERA DE LA RED, Micaela (1998) : "Grafías y grafemas representativos de sibilantes en documentos dominicanos de los siglos XVI y XVII. Propuesta de análisis», in José Manuel Blecua, Juan Gutiérrez \& Lidia Sala (éds). Estudios de grafemática en el dominio hispano. Salamanca, Universidad de Salamanca / Instituto Caro y Cuervo, 25-35.

CATACH, Nina (1968) : L'orthographe française à l'époque de la Renaissance (Auteurs, Imprimeurs, Ateliers d'imprimerie). Genève, Droz.

CATACH, Nina (1977). «La ponctuation dans les imprimés, des débuts de l'imprimerie à G. Tory et E. Dolet », in Nina Catach \& Claude Tournier (éds), La ponctuation. Recherches historiques et actuelles. Vol. 1 : Documents préparatoires à la Table ronde internationale CNRS, mai 1978, Paris / Besançon, CNRS-GTM-HESO, 29-57.

CATACH, Nina (1980). "La ponctuation ». Langue Française, 45 [Nina Catach (éd.) La ponctuation], 16-27.

CATACH, Nina [éd.] (1988) : Pour une théorie de la langue écrite. Paris, CNRS.

CATACH, Nina (1994) : La ponctuation (histoire et système). Paris, Presses universitaires de France (coll. "Que sais-je?»).

CATACH, Nina (1995a) : L'orthographe française. Traité théorique et pratique avec des travaux d'application et leurs corrigés. $3^{\mathrm{e}}$ éd., Paris, Nathan.

CATACH, Nina [dir.] (1995b) : Dictionnaire historique de l'orthographe française (DHOF). Paris, Larousse.

CATACH, Nina (1998) : «Les signes graphiques du mot à travers l'histoire ». Langue Française, 119 [N. Andrieux-Reix, et S. Monsonégo (éds) Segments graphiques du français. Pratiques et normalisations dans l'histoire], 10-23.

CATACH, Nina (2001a) : Histoire de l'orthographe française. Édition posthume de Renée Honvault et Irène Rosier-Catach. Paris, Champion. 
CATACH, Nina (2001b) : "Graphétique et graphémique ", in G. Holtus, M. Metzeltin, C. Schmitt (éds), Lexikon der Romanistischen Linguisik, 1, II. Mëthodologie, chap. 2627, Tübingen, Niemeyer, 725-748.

CATACH, Nina, Jeanne GOLFAND \& Roger DENUX (1971): Orthographe et lexicographie (Littré, Robert, Larousse). Tome I, Paris, Didier.

CATACH, Nina \& Claude TOURNIER [éds] (1977) : La ponctuation. Recherches historiques et actuelles. Vol. 1 : Documents préparatoires à la Table ronde internationale CNRS, mai 1978, Paris / Besançon, CNRS-GTM-HESO.

CATACH, Nina \& Jacques PETIT [éds] (1979) : La ponctuation. Recherches historiques et actuelles. Vol. 2 : Actes de la Table ronde internationale CNRS, mai 1978. Paris / Besançon, CNRS-GTM-HESO.

Cerquiglini, Bernard (1989) : Éloge de la variante. Histoire critique de la philologie. Paris, Seuil.

CERQUIGLINI, Bernard (1991) : La naissance du français. Paris, Presses universitaires de France.

CERQUiglini, Bernard (1995) : L'accent du souvenir. Paris, Minuit.

CERQUIGLINI, Bernard (1996): Le roman de l'orthographe. Au paradis des mots avant la faute. 1150-1694. Paris, Hatier.

CERQUiglini, Bernard (2004) : La Genèse de l'orthographe française (XII'-XVII siècles). Paris / Genève, Champion / Slatkine.

CERQUiglini, Bernard (2007) : Une langue orpheline. Paris, Minuit.

CERQUiglini, Bernard (2018) : L'invention de Nithard. Paris, Minuit.

COlOMBO, Maria (2008) : "La punteggiatura in Francia. II. Dal XIV secolo a oggi ", in Bice Mortara Garavelli (dir.), Storia della punteggiatura in Europa. Roma, Barbi, 233-293.

COMBETTES, Bernard (2012) : "Linguistique textuelle et diachronie ", in Actes du $3^{e}$ Congrès Mondial de Linguistique Française, EDP Sciences, 3-10, DOI : https://doi.org/$10.1051 /$ shsconf/20120100344

COMBETTES, Bernard (2019) : "Aspects de l'évolution de la cohérence textuelle et discursive ", in Daniéla Capin, Julie Glikman, Vanessa Obry \& Thierry Revol (éds). Le français en diachronie (Moyen français - Segmentation des énoncés - Linguistique textuelle). Strasbourg, EliPhi, Éditions de Linguistique et de Philologie, 153-168.

COMBETTES, Bernard (2020) : «Partie 8. Énonciation et textualité, pragmatique. Introduction : Évolutions dans le domaine de la cohérence discursive » et "Chapitre 43. Niveau informationnel », in GGHF, 1697-1701 et1739-1777.

CONDORELLI, Marco [éd] (2020) : Advances in Historical Orthography, c. 1500-1800, Cambridge / New York, Cambridge University Press.

CONDORELlI, Marco \& Anja VOeste (2020): «Synergic Dialogue in Historical Orthography. National Philologies Comparability and Questions for the Future», in Marco Condorelli (éd), Advances in Historical Orthography, c. 1500-1800, Cambridge / New York, Cambridge University Press, 238-249. 
CONDORELLI, Marco [éd] (à paraitre). The Handbook of Historical Orthographies. Cambridge / New York, Cambridge University Press.

DAHLET, Véronique (2003) : Ponctuation et énonciation. Guadeloupe, Ibis Rouge Éditions.

DAUVOIS, Nathalie \& Jacques DÜRRENMATT [éds] (2011) : La Ponctuation à la Renaissance. Paris, Garnier.

DAVID, Jacques \& Sandrine VAUDREY-LUIGI [éds] (2014) : Le français aujourd'hui, $187: 4$. URL : https://www.revues.armand-colin.com/lettres-langue/francais-aujourdhui

DÜRRENMATT, Jacques [éd.] (2000) : La ponctuation. Poitiers, UFR Langues, Littératures (coll. « La licorne », 52).

FASSEUR, Valérie \& Cécile ROCHELOIS [éds] (2016) : Ponctuer l'auvre médiévale. Des signes au sens. Genève, Droz.

FAVRIAUD, Michel [éd.] (2011) : Langue française, 172 [Ponctuation(s) et architecturation $d u$ discours à l'écrit].

FERrARI, Angela, Letizia LALA \& Filippo PECORARI (2017) : L'interpunzione oggi (e ieri). L'italiano e altre lingue europee. Firenze, Franco Cesati Editore.

GGHF = MARCHELlo-Nizia, Christiane ; Bernard CombetTes ; Sophie PréVOST \& Tobias SCHEER [éds] (2020) : Grande Grammaire Historique du Français (GGHF), 2 vols., Berlin / Munich / Boston, Walter de Gruyter GmbH.

Las Heras Calvo, Miguel (2020) : La puntuación sintáctica en la General estoria de Alfonso $X$ el Sabio. Thèse Doctorale, Universidad de la Rioja.

LAVRENTIEV, Alexei (2009): Tendances de la ponctuation dans les manuscrits et incunables français en prose, $d u X I I I^{e}$ au XVe siècle. Thèse doctorale, 2 vols. Lyon, ENS-LSH, URL : https://tel.archives-ouvertes.fr/tel-00494914

LLAMAS-POMBO, Elena (1996) : "Contribución a la historia de la puntuación medieval francesa ", Escritura y oralidad en los Ovidiana franceses del siglo XII. Thèse doctorale sous la direction de Isabel Uzcanga Vivar. Salamanque, Université de Salamanque, 241407.

LLAMAS-POMBO, Elena (2008) : «Ponctuer, éditer, lire. État des études sur la ponctuation dans le livre manuscrit ". Syntagma. Revista del Instituto de Historia del Libro y de la Lectura 2, 129-171.

LLAMAS-POMBO, Elena (2009): «Variación gráfica y secuenciación de la palabra en manuscritos medievales hispánicos», in Pedro M. Cátedra (dir); Eva Belén Carro Carbajal \& Javier Durán Barceló (éds), Los códices literarios de la Edad Media. Interpretación, historia, técnicas y catalogación, San Millán de la Cogolla, Cilengua, 225-257. URL: https:/gredos.usal.es/jspui/handle/10366/122006

LLAMAS-POMBO, Elena (2016). "Ponctuation médiévale, pragmatique et énonciation. Lire l'Ovide Moralisé au XIV siècle ". Linx. Revue des linguistes de l'Université Paris Nanterre, 73 (Amalia Rodríguez Somolinos [éd.], Énonciation et marques d'oralité dans l'évolution du français), 113-145. 
LlamaS-POMBO, Elena (2020a) : "Punctuation in Sixteenth- and Seventeenth-Century French and Spanish Grammars. A Model of Diachronic and Comparative Graphematics ", in Marco Condorelli (éd.), Advances in Historical Orthography, c. 1500-1800, Cambridge / New York, Cambridge University Press, 93-123.

LlaMAS-POMBO, Elena (2020b) : "Chapitre 18. Ponctuation », in GGHF, 592-614.

MARCHELLO-NIZIA, Christiane (2014) : "L'importance spécifique de l'oral représenté pour la linguistique diachronique ", in Wendy Ayres-Bennett \& Thomas M. Rainsford, L'histoire du français. État des lieux et perspectives. Paris, Classiques Garnier, 161-174.

MAZZIOTTA, Nicolas (2009) : Ponctuation et syntaxe dans la langue française médiévale. Étude d'un corpus de chartes originales écrites à Liège entre 1236 et 1291. Tübingen, Max Niemeyer.

MESCHONNIC, Henri (2000) : "La ponctuation, graphie du temps et de la voix », in Jacques Dürrenmatt (éd.), La ponctuation. Poitiers, UFR Langues, Littératures (coll. « La Licorne ", 52), 289-293.

PARUSSA, Gabriella \& Yvonne CAZAL (2020) : "Codes de l'écrit : Graphies et ponctuation ", in GGHF, 491-614.

PARUSSA, Gabriella; Maria COLOMBO \& Elena LlAMAS-POMBO [éds] (2017) : "Introduction ", Enregistrer la parole et écrire la langue dans la diachronie du français. Tübingen, Günter Narr (coll. "ScriptOralia », 143).

PETILlon, Sabine [éd.] (2004) : L'information grammaticale, 102 [La ponctuation].

PÉTILlON, Sabine ; Fanny RINCK \& Antoine GAUTIER [éds] (2016) : La Ponctuation à l'aube du XXI' siècle. Perspectives historiques et usages contemporains. Actes des Journées d'études de Nanterre du 29 mars 2013 et du 4 avril 2014. Limoges, Lambert-Lucas.

RAIBLE, Wolfgang (1992) : Junktion: Eine Dimension des Sprache und ihre Realisierungsformen zwischen Aggregation und Integration. Heidelberg, Winter (Sitzungsberichte der Heidelberger Akademie der Wissenschaften, Berichtheft, 2).

SÁNCHEZ-PRIETO BORJA, Pedro (2012) : «Para una historia de la escritura romance en León, Castilla y Aragón: algunas claves interpretativas ». Medioevo romanzo, 36, 24-61.

SCHNEDECKER, Catherine (1997) : Nom propre et chaînes de référence. Paris, Klincksieck.

SCHNEDECKER, Catherine (2017) : "Les chaînes de référence : une configuration d'indices pour distinguer et identifier les genres textuels ». Langue française, 195, 53-72.

SCHNEDECKER, Catherine \& Frédéric LANDRAGIN (2014) : "Les chaînes de référence : présentation ». Langages, 195, 3-22.

SCHNEDECKER, Catherine; Julie GLiKMAN \& Frédéric LANDRAGIN [éds] (2017) : Langue française, 195 [Les chaînes de référence en corpus]. 


\section{Post scriptum}

Pendant l'élaboration de ce numéro monographique, l'Université de Salamanque a perdu l'une de ses professeures au Département de Philologie Française : María Teresa Lozano Sampedro. Elle a consacré une longue vie professionnelle à l'enseignement de la littérature française en Espagne, enseignement qui demeurera vivant chez les étudiants qui ont suivi ses cours pendant quatre décennies. Nous lui rendons hommage ; qu'elle repose en paix.

Elena Llamas-Pombo \& Diana Gatea Universidad de Salamanca 